La FMH s'engage en faveur d'une haute qualité dans la santé et d'une concurrence équitable. En matière de qualité, cela est seulement possible si les données qualité sont relevées et évaluées correctement. Une publication de ces données implique de suivre les recommandations de l'Académie Suisse des Sciences Médicales (ASSM) afin d'éviter qu'il y ait plus de dommages que d'avantages. La priorité doit toujours être donnée aux besoins des patientes et des patients. C'est pourquoi la FMH soutient une transparence vis-à-vis des groupes cibles. En effet, les différents groupes concernés n'ont pas la même utilité ni la même interprétation des données qualité. De plus, toutes les données qualité ne sont pas aptes à être publiées à grande échelle. Des données incomplètes, fausses ou inexactement analysées ou publiées déforment la réalité et empêchent le corps médical, les hôpitaux et les cliniques de montrer la bonne qualité de leurs prestations. Ces mauvais incitatifs mènent inévitablement à des évolutions dramatiques, ce qui se traduit par une baisse de qualité. La population est ainsi également induite en erreur et sa confiance envers le système de santé injustement affaiblie. Un effet positif, induit par de bons incitatifs, ne peut être obtenu que par la publication de données qualité complètes, correctes et compréhensibles.

\title{
Plaidoyer pour des comparaisons équitables entre hôpitaux et cliniques
}

\section{Petra Busch}

Dr rer. pol., directrice de I'ANO

\section{Des informations claires et concises sur les prestations des hôpitaux, des cliniques} et du corps médical sont de plus en plus nécessaires. Ce besoin fait naître des portails de recherche et de comparaison d'établissements qui, en Suisse, se réfèrent tous aux résultats des mesures de l'ANQ; certains en tirent même des classements. Mais l'ANQ rejette fermement ces derniers, car ils sont scientifiquement impossibles.

Depuis 2009, l'ANQ coordonne et réalise, dans l'ensemble de la Suisse, des mesures de la qualité uniformes portant sur les hospitalisations en médecine somatique aiguë, en réadaptation et en psychiatrie. Elle applique ainsi les prescriptions de la Loi sur l'assurance-maladie (LAMal). Etant, dans son activité, tenue à la plus grande attention et transparence scientifiques, elle présente et communique en conséquence les résultats de ses mesures, qui sont toujours situés dans le contexte général, et accompagnés de graphiques clairs et d'aides à la lecture.

Lorsqu'il s'agit de communiquer les résultats, les comparaisons entre les hôpitaux et les cliniques doivent absolument être équitables. L’ANQ, amenée de ce fait à suivre l'évolution des portails de recherche et de comparaison des hôpitaux, désapprouve l'utilisation par des tiers non autorisée, réduite ou inappropriée des résultats de ses mesures. En particulier, ces résultats ne conviennent pas pour classer les hôpitaux et les cliniques, car une telle démarche est impossible pour plusieurs raisons.

\section{Qu'est-ce qu'un indicateur de qualité?}

Avant de réaliser une mesure, il faut définir quel est l'indicateur de qualité approprié. Les indicateurs de qualité sont des outils servant à l'évaluation quantitative de fonctions importantes qui influent sur le résultat du traitement des patientes et des patients. Ils peuvent refléter la qualité des structures, des processus ou des résultats qui sont essentiels pour le monitorage et la gestion de la qualité. Ils aident ainsi à 
mieux percevoir les domaines potentiellement problématiques, obligent à les analyser, à les contrôler et à les améliorer. Les chiffres obtenus peuvent se situer à l'intérieur ou à l'extérieur du domaine de référence, ou bien au-dessus ou au-dessous d'une valeur de référence. Mais un indicateur n'est pas une mesure directe de la qualité.

\section{Des aspects différents pour une qualité globale}

Les scientifiques discutent, sur le plan national comme sur le plan international, pour savoir si un indicateur de qualité peut être employé en dehors de son domaine habituel d'utilisation, par exemple pour la planification hospitalière ou le paiement à la performance (rémunération en fonction de la qualité). Mais le fait est là: il n'existe pas d'indicateur polyvalent, capable de donner une indication claire et nette quant à la qualité globale d'un hôpital ou d'une clinique - et, en l'état actuel des connaissances, un tel indicateur n'est pas près d'exister. Par conséquent, chaque résultat de mesure de l'ANQ ne reflète qu'un certain aspect de la qualité d'un établissement, dans le cadre d'une certaine mesure. Extrapoler ce résultat à la qualité globale d'un hôpital ou d'une clinique n'est pas admissible, pas plus que présenter cette image aux utilisateurs d'Internet en publiant une telle évaluation.

\section{Opportunités offertes par les indicateurs de l'ANQ}

Les indicateurs que l'ANQ a choisis sont principalement adaptés au développement de la qualité dans les hôpitaux et les cliniques suisses, à qui ils fournissent une base sur laquelle définir des améliorations ciblées et les mettre en œuvre. Ils conviennent pour des mesures identiques dans l'ensemble du pays, ainsi que pour l'information transparente et la publication des résultats - mais à condition que les données soient d'une qualité suffisante. Ils permettent une comparaison équitable des résultats entre les hôpitaux et les cliniques, ainsi qu'avec le résultat global de tous les établissements. Les cantons et les assureurs s'en servent comme point de départ pour le dialogue avec ces derniers.

\section{Limites des indicateurs de la qualité de l'ANQ}

Les résultats des mesures de l'ANQ ne peuvent servir ni à l'allocation des ressources, ni au paiement à la performance; il faudrait pour cela définir d'autres indica- teurs. L'ANQ déconseille aussi formellement de les détourner de leur objectif pour sanctionner les hôpitaux et les cliniques qui ont des résultats inférieurs à la moyenne. En effet, selon le domaine, l'indicateur de qualité choisi et l'instrument de mesure qui y est associé, la significativité de chaque mesure est limitée. A chacune d'entre elles sont liées des opportunités et des limites spécifiques, et en particulier des risques de mauvaise interprétation, comme le montre l'exemple suivant.

\section{Exemple: taux d'infections du site chirurgical après une intervention}

Swissnoso, l'association des principaux spécialistes dans le domaine des maladies infectieuses et de l'hygiène hospitalière, surveille depuis huit ans, à la demande de l'ANQ, l'évolution des infections postopératoires avec un instrument appelé Surveillance des SSI. Comme, de manière générale, les infections hospitalières, en particulier celles qui surviennent après une intervention, sont en rapport avec la qualité des prestations d'un hôpital ou d'une clinique, la publication transparente des taux d'infections postopératoires intéresse beaucoup la population et les médias.

Pour interpréter et communiquer les résultats, il faut toutefois tenir compte de plusieurs facteurs et éliminer ceux qui seraient susceptibles de donner une fausse image de la situation:

- Les infections postopératoires doivent toujours être considérées dans le contexte d'une intervention chirurgicale. Même suivie d'une infection, une opération peut atteindre son but, par exemple soulager des douleurs ou améliorer le fonctionnement d'une articulation. Il n'est de toute façon pas possible d'éviter totalement les infections après des interventions; en outre, certaines sont plus susceptibles de s'infecter du simple fait de leur localisation, par exemple le côlon ou le rectum.

- La méthode statistique d'ajustement des risques ${ }^{1}$ n'est pas parfaite et ne peut pas corriger toutes les différences entre les établissements.

- La qualité du relevé des données est calculée à l'aide d'instruments standardisés (validation). Les taux d'infections pouvant être biaisés pour les hôpitaux et les cliniques où la qualité du relevé est insuffisante, ils doivent être interprétés avec prudence.

- Les taux d'infections suisses ne sont pas tout à fait comparables à ceux des systèmes de surveillance d'autres pays. Il existe des différences parfois déter-

L'ANO traite de ce thème pour chaque mesure et chaque indicateur dans son argumentaire "Opportunités et limites des mesures de la qualité de I'ANQ»: www.anq.ch/fr/resultats-de-mesure 
minantes en ce qui concerne la méthode et la qualité du relevé, ainsi que la surveillance après la sortie. Comme, de manière générale, la Suisse privilégie des relevés plus rigoureux et une surveillance plus longue, elle présente parfois des taux plus élevés que les autres pays.

\section{Effets positifs}

Les mesures de la qualité de l'ANQ donnent aux hôpitaux et aux cliniques des informations précises leur permettant de se comparer aux autres. Facilement mises en œuvre, elles présentent un intérêt pratique pour leur travail quotidien. Souvent, leurs résultats déclenchent des processus d'amélioration et fournissent des arguments en faveur des investissements correspondants: analyses de processus, formations, mise au point de stratégies, etc. Au sein de l'hôpital ou de la clinique, la publication transparente a des répercussions à plusieurs niveaux: elle aide à établir des programmes, sensibilise, met en évidence les points où des changements s'imposent, et peut même entraîner une évolution dans les habitudes de pensée et de travail.

Correspondance:

ANQ, Association nationale pour le développement de la qualité dans les hôpitaux

et les cliniques

Thunstrasse 17

Case postale 370

CH-3000 Berne 6

Tél. 0315113840

petra.busch[at]anq.ch

www.anq.ch

\section{Dialoguer plutôt que mettre au pilori}

L'ANQ apprécie que l'on publie les résultats de ses mesures de la qualité et que l'on en discute de manière objective. Elle apporte ainsi une contribution importante à l'indispensable discussion sur la qualité au sein du système suisse de santé.

L'ANQ comprend très bien qu'il est de plus en plus nécessaire de disposer d'informations claires sur la
L'Association nationale pour le développement de la qualité dans les hôpitaux et les cliniques (ANQ)

L'Association nationale pour le développement de la qualité dans les hôpitaux et les cliniques (ANQ) est une association sans but lucratif qui coordonne et réalise des mesures de la qualité en médecine somatique aiguë, en réadaptation et en psychiatrie. Les résultats permettent une comparaison transparente et nationale. A partir de ces résultats, les hôpitaux et les cliniques peuvent développer des mesures ciblées pour améliorer leur qualité. Ses membres sont $\mathrm{H}+$ Les hôpitaux de Suisse, santésuisse, les assurances sociales fédérales, les cantons et la Conférence suisse des directeurs et directrices cantonaux de la santé.

www.anq.ch

qualité. Mais, pour les raisons citées plus haut, de simples classements non seulement ne servent pas le développement de la qualité, mais risquent même d'être contre-productifs. L'ANQ ne met donc aucun hôpital en avant, ni dans un sens positif ni dans un sens négatif. La simple publication transparente des résultats oblige ceux qui ont des mauvais chiffres à réagir. Grâce aux données uniformes et donc comparables fournies par les mesures, ils ont la possibilité de mettre le doigt sur le problème et de viser le meilleur pour leur type d'hôpital ou de clinique. 